\title{
Socialization of Pain Memories: Parent-Child Reminiscing About Past Painful and Sad Events
}

\author{
Maria Pavlova, ${ }^{1}$ MSc, Susan A. Graham, ${ }^{1,2}$ PHD, Abbie Jordan, $^{3}$ PHD, \\ Jill Chorney, ${ }^{4}$ PHD, Jillian Vinall, ${ }^{5}$ PHD, Nivez Rasic, ${ }^{5}$ MD, \\ James Brookes, ${ }^{6}$ MD, Monica Hoy, ${ }^{6}$ MD, Warren K. Yunker, ${ }^{6}$ MD, PHD, \\ and Melanie Noel, ${ }^{1,7}$ PHD
}

\footnotetext{
${ }^{1}$ Department of Psychology, University of Calgary, ${ }^{2}$ Owerko Centre, ${ }^{3}$ Department of Psychology, University of Bath, ${ }^{4}$ Department of Anesthesia, Pain Management and Perioperative Medicine, Dalhousie University, ${ }^{5}$ Department of Anesthesia, University of Calgary, ${ }^{6}$ Department of Surgery, University of Calgary, and ${ }^{7}$ Alberta Children's Hospital Research Institute
}

All correspondence concerning this article should be addressed to Maria Pavlova, MSc, Department of Psychology, The University of Calgary, 2500 University Dr. N.W. Calgary, AB T2N 1N4, Canada. E-mail: mpavlova@ucalgary.ca

Received September 25, 2018; revisions received January 20, 2019; accepted January 30, 2019

\begin{abstract}
Objective Parent-child reminiscing about past negative events has been linked to a host of developmental outcomes. Previous research has identified two distinct between-parent reminiscing styles, wherein parents who are more elaborative (vs. repetitive) have children with more optimal outcomes. To date, however, research has not examined how parents and children talk about past painful experiences nor compared parent-child reminiscing about past painful versus other distressing events despite key developmental differences in how young children respond to pain versus sadness in others. This study aimed to fill that gap. Methods Seventy-eight children aged 4 to 7 years underwent a tonsillectomy. Two weeks postsurgery, children and one of their parents discussed past autobiographical events (i.e., the tonsillectomy, another painful event, a sad event). Parent-child conversations were coded using established coding schemes to capture parental reminiscing style, content, and autonomy support. Results Findings revealed robust differences in parent-child reminiscing about painful versus sad events. Parents were less elaborative, used less emotionally negative words and explanations, and were less supportive of their children's autonomy while reminiscing about past painful versus sad events. Conclusions These findings demonstrate that through reminiscing, parents may socialize children about pain in a way that is different from other distressing events (e.g., sadness). Future research should examine the influence of differential reminiscing about pain versus sadness on developmental and health outcomes.
\end{abstract}

Key words: children; pain; parents; reminiscing; sadness.

\section{Introduction}

Parent-child reminiscing powerfully shapes young children's cognitive and socioemotional development by creating a foundational learning framework that helps children to process and assign meaning to their past distressing experiences (Nelson \& Fivush, 2004). Through elaborative and supportive dialogs enriched with emotional language, children and parents cocreate coherent narratives about past distressing events, which, in turn, lead to children internalizing 
adaptive ways of processing distressing emotions (Fivush, Haden, \& Reese, 2006). However, parentchild conversations about pain, a universal distressing sensory and emotional experience, and specifically past pain, have not been examined.

Past painful experiences are stressful, salient, and memorable. An investigation of children's memories for painful injuries that required a visit to the Emergency Department revealed that children as young as 3 years recalled many details of their injuries up to 10 years later (Peterson, 2015). Further, children's memories for pain are a more robust predictor of future pain than the initial reporting of pain (Noel, Chambers, McGrath, Klein, \& Stewart, 2012). That is, young children with negatively biased memories for pain (i.e., recall of more pain as compared with initial reports) reported more pain during a future experimental pain task (Noel et al., 2012). Children's negatively biased pain memories postsurgery have been linked to higher pain at the time that it can transition to a chronic state (Noel, Rabbitts, Fales, Chorney, \& Palermo, 2017). These pain memories are thought, in part, to be (re)constructed through parent-child verbal interactions following painful events (Noel, Palermo, Chambers, Taddio, \& Hermann, 2015). Yet, research on parentchild reminiscing about painful events is scarce.

In addition to pain, children regularly experience other distressing events that are associated with interpersonal sadness and distress (e.g., separation from caregivers). These painful and distressing experiences are memorable and often become the topic of parentchild conversations (Laible \& Song, 2006). During reminiscing, parents and children coconstruct children's memories, which then influences children's responses in similar situations in the future (Salmon $\&$ Reese, 2015, 2016). Indeed, research in developmental psychology has long established that parent-child reminiscing, particularly reminiscing about past negative autobiographical events, has a powerful influence on children's cognitive (attention, memory, language, and theory of mind), social (empathy), and emotional (emotion regulation) development (Leyva, Berrocal, \& Nolivos, 2014; Leyva \& Nolivos, 2015; Salmon \& Reese, 2015, 2016).

The ways in which parents reminisce with children about past events vary markedly across parents. These differences in reminiscing have important implications for children's outcomes. While reminiscing, some parents are elaborative, in that that they use openended questions and emotion words and provide rich contextual details (Fivush \& Fromhoff, 1988). Further, parents who use an elaborative reminiscing style tend to support children's conversational contributions by following in on children's statements, thus creating a more comprehensive memory account and providing validation (Cleveland \& Morris, 2014;
Cleveland \& Reese, 2005). An elaborative reminiscing style has been linked to beneficial developmental outcomes, including more accurate and earlier autobiographical memories (Reese \& Robertson, 2019; Salmon \& Reese, 2015) and empathic prosocial responding (Laible \& Song, 2006; Leyva et al., 2014). Conversely, parents who use a nonelaborative/repetitive reminiscing style use closed-ended questions and frequently switch conversation topics without providing additional contextual details (Sales, Fivush, \& Peterson, 2003). These parents do not discuss emotions or support children's autonomy during these conversational exchanges. This reminiscing style is linked to poorer developmental outcomes. Specifically, children, whose parents provided less autonomy support, elaborations, and emotional language, recalled less unique details about past autobiographical events (Cleveland \& Reese, 2005; Sales et al., 2003), demonstrated worse emotional understanding and prosocial behaviors (Laible, 2004).

Little is known about how different types of distressing events may pull for different or particular styles of reminiscing within parents. Fivush, Berlin, Sales, Mennuti-Washburn, \& Cassidy (2003) compared parental reminiscing styles across three types of negative events (i.e., fear, sadness, and anger). Fear narratives were found to be more elaborative as compared with narratives about sadness and anger. However, no studies to date have examined differences between parent-child reminiscing about past painful events versus other types of negative events (e.g., events involving sadness). This question is relevant to the field of pediatric pain as developmental differences in young children's empathic behavioral responses (e.g., distracting the other person, providing verbal reassurance/sympathy) to pain versus sadness in others have been found (Bandstra, Chambers, McGrath, \& Moore, 2011). When exposed to behavioral distress in an adult that originated from sadness or pain, children were more distressed by and empathically responsive to sadness versus pain (Bandstra et al., 2011). Individual difference variables (e.g., children's age, negative affectivity, and language) were predictive of some variance $(8-13 \%)$ in children's empathic concern and personal distress related to pain in others (Bandstra et al., 2011). Yet, a large proportion of variance remained unexplained. This raises the possibility that the differences in children's prosocial responding to pain versus sadness in others may be partially driven by differential socialization of pain versus negative emotions, like sadness, and parent-child interactions.

This study addressed this gap by examining the reminiscing style and content across parent-child reminiscing about two painful autobiographical events (a recent surgery and another painful event) and a 
negative emotional autobiographical event (a sad event). Based on the differences in reminiscing styles that were revealed as a function of the reminiscing topic (Fivush, Berlin, et al., 2003) and the developmental differences found between young children's behavioral responses to pain versus sadness (Bandstra et al., 2011), we hypothesized that parents and children would reminisce differently when talking about a past event involving sadness as compared with pain. Given the dearth of research on parent-child reminiscing about past pain, we did not have specific directional hypotheses about the differences between reminiscing styles as a function of the event type.

\section{Method}

\section{Participants}

Seventy-eight children aged 4-7years $\left(M_{\text {age }}=\right.$ 5.21 years, $S D=1.06,42 \%$ girls) scheduled to undergo a tonsillectomy with or without adenoidectomy and one of their parents (73\% mothers) were recruited a tertiary children's hospital in Western Canada (see Table 1 for the sociodemographic characteristics of the sample). The institution's health research ethics board approved this study. Consistent with previous research (Kain, Mayes, Caldwell-Andrews, Karas, \& McClain, 2006), children were excluded if they received premedication with anxiolytics (midazolam) and/or had serious medical comorbidities or developmental disabilities. Approximately $80 \%$ of families who were contacted agreed to participate; $83 \%$ of recruited families completed all stages of the study $(N=81)$, and three families did not generate a past sad event and, thus, were excluded from analyses.

\section{Measures}

\section{Demographics}

Parents reported sociodemographic information.

Pain Intensity, Pain-Related Fear, and Negative Affect The Faces Pain Scale-Revised (FPS-R) is a wellvalidated and reliable self-report measure of pain intensity in children 4-16 years old (Hicks, von Baeyer, Spafford, van Korlaar, \& Goodenough, 2001). Six faces vary in expression of pain ranging from a neutral facial expression (0) up to a face in extreme pain (10). Prompted by the researcher, children point to the face that reflects how much they hurt. Children used the FPS-R to rate their pain intensity $2-3 \mathrm{hr}$ postsurgery and on the first day after the surgery as well as their recalled pain intensity associated with the postsurgical period (i.e., the first day and first few days after the surgery) and the nonsurgery painful event. The Children's Fear Scale (CFS) was used to assess children's pain-related fear $2-3 \mathrm{hr}$ postsurgery and on the first day after the surgery as well as their recalled
Table I. Participant Sociodemographic Characteristics.

\begin{tabular}{lc}
\hline Demographic characteristic & $N=78$ \\
\hline Age, $M(S D)$ & $5.21(1.06)$ \\
Sex (female) $(\%)$ & 42 \\
Ethnicity (\%) & \\
Aboriginal & 1 \\
Arab/West Asian & 1 \\
Black & 2 \\
Filipino & 4 \\
South Asian & 2 \\
White & 84 \\
Other & 2 \\
Multiple ethnicity & 4 \\
Participating parent (mother) (\%) & 73 \\
Parent highest level of education $(\%)$ & \\
High school or less & 10 \\
Vocational school/some college & 30 \\
College degree & 48 \\
Graduate school & 12 \\
Work status (\%) & \\
Full time & 51 \\
Part time & 22 \\
Not working & 27 \\
Household annual income (\%) & \\
Less than \$10,000 & \\
\$10,000-\$19,000 & \\
\$20,000-\$29,000 & \\
\$40,000-\$49,000 & \\
\$50,000-\$59,000 & 4 \\
\$60,000-\$69,000 & 4 \\
More than \$70,000 & 4 \\
\hline
\end{tabular}

pain-related fear associated with the postsurgical period and the nonsurgery painful event (McMurtry, Noel, Chambers, \& McGrath, 2011). This scale depicts five faces of varying degrees of fear with anchors 0 (not at all scared) to 4 (most scared possi$b l e)$. It shows evidence of good concurrent and discriminant validity and test-retest reliability in children as young as 5 years (McMurtry et al., 2011) and has been used for pain-related fear assessment in children aged 2 years and older (Hyland et al., 2015). In McMurtry et al.'s study (2011), children used the FPS$\mathrm{R}$ and CFS scales to provide self-report of pain and pain-related fear during a venipuncture. The scales' ratings were moderately correlated $(r=.65)$, yet, the association was in line with previous research on children's ratings of pain and pain-related constructs. Finally, a faces scale representing emotions and ranging from a sad face (1) to a happy face (7) was used during the laboratory visit to assess children's recalled emotional affect associated with the sad autobiographical event (Castilla, Botella, García-Palacios, Farfallini, \& Miralles, 2012). Children's ratings of emotional affect associated with particular past events is not typically assessed. The emotion faces scale's ratings were intended only to descriptively assess the degree of children's perceived emotional valence of the sad event and were not used in the subsequent analyses. Parents rated how much they had previously 
talked with their children about each autobiographical event on an 11-point Likert scale [anchors: 0 (not at all) to $10($ a lot $)]$.

\section{Procedure}

Approximately 1-3 weeks before surgery, parents were contacted via telephone and interested eligible families were sent an email link via REDCap, a secure online database, to the consent forms and the demographic questionnaire. On the day of the surgery, all patients received paracetamol $15 \mathrm{mg} / \mathrm{kg}$ orally preoperatively and an inhalational induction with sevoflurane, oxygen, and nitrous oxide. During the procedure, patients received dexamethasone $0.2 \mathrm{mg} / \mathrm{kg} \mathrm{IV}$, ondansetron $0.1 \mathrm{mg} / \mathrm{kg} \mathrm{IV}$, and morphine for analgesia. Surgeons used cautery to the tonsillar bed as their surgical technique. Patients recovered in the postanesthesia care unit, and when conscious/stable they returned to the day surgery unit until discharge. The FPS-R and the CFS were administered by the research staff $2-3 \mathrm{hr}$ after the surgery (Timepoint 1$)$. In line with previous research (MacLaren \& Kain, 2008), parents observed the scale administration and received verbal and written instructions on how to use the scales to assess children's pain intensity and painrelated fear on the first day after the surgery (Timepoint 2).

Two weeks following the tonsillectomy, parents and children returned to the hospital for a laboratory visit. At this time, parents and children engaged in a structured narrative elicitation task (Salmon \& Reese, 2015), which prompted them to reminisce with one another about three autobiographical events: the child's recent tonsillectomy surgery, another past painful experience, and an event involving sadness. A past painful experience narrative was elicited to examine potential differences between the unique tonsillectomy surgery (which by nature can only occur once) and nonsurgery painful events (which were often everyday pain events that could have occurred repeatedly). Indeed, differences in recall between unique versus repeated autobiographical events have been found (Powell \& Thomson, 1996). The order of discussed events remained the same across the dyads (i.e., the recent surgery, another painful event, and a sad event). To ensure participants did not leave in a state of distress as a result of reminiscing about negative events, the laboratory visit was concluded by reminiscing about a past event involving happiness. The latter was not relevant to the aims of the current study and was not included in the analyses. The elicited events were experienced/shared by both the parent and the child, and there was no time limit for parents and children to reminisce (Sales \& Fivush, 2005). Parents and children were instructed to talk as they normally would at home; the researcher left the room for the duration of the task (Valentino et al., 2015). After the task, the research staff administered the FPS-R and the CFS to assess the recalled pain and pain-related fear associated with everyday painful events, and the emotion scale to assess affect associated with the sad event. The recalled postsurgical pain and pain-related fear were not assessed. Parents rated the extent to which they talked with their child about each event prior to the laboratory visit on an 11-point Likert scale.

\section{Narrative Coding Scheme}

All parent-child narratives were audio- and videorecorded, transcribed verbatim, and broken down into utterances, which were defined as a conversational turn. Utterances were coded using a coding scheme drawn from the child development literature (Sales et al., 2003). Specifically, parent utterances were coded for three aspects: structure, content, and autonomy support style (see Supplementary material for examples of each code). To control for differences in the length of narratives and in line with previous research (Sales et al., 2003), proportions for each code over the total number of codes used were calculated for each code type. Means and standard deviations for each event and utterance type are presented in Table 2. Two coders were trained on the coding scheme by concurrently coding five transcripts. After discussing all discrepancies, one of the coders coded the remaining transcripts. Twenty percent of the transcripts (15) were independently coded by two coders. Coders achieved $\geq .80$ reliability across structure and content codes and .71 across autonomy support codes (Cohen's kappa).

\section{Structure}

Structural coding was based on the research of Sales and colleagues (2003). Each parent sentence was coded as either a question or a statement. Questions were further coded as closed (e.g., yes-no questions) or open-ended and as containing new (i.e., elaborative) or old (i.e., repetitive) information. Similarly, statements (i.e., sentences that did not require an answer from the child) were coded as those containing new (i.e., elaboration) or old (i.e., repetition) information. In addition, one-word sentences (e.g., "Yeah", "No") were coded as evaluations. Codes were assigned to child utterances depending on whether they contained novel information (i.e., memory elaboration) or were a one-word reply (memory placeholder; e.g., "Yes", "No", "I don't know"). If a child started an utterance by talking about a nonrelated topic, an off-topic code was assigned, and further utterances were not coded. We expected that parents would use elaboration codes (memory question elaboration [MQE], yes-no question elaboration [YNE], and statement elaboration [SE]) and repetition codes 
Table II. Mean Proportions and Standard Deviations for Parental and Child Narrative Codes

\begin{tabular}{|c|c|c|c|c|c|c|}
\hline \multirow[b]{3}{*}{ Code type } & \multicolumn{6}{|c|}{ Event type } \\
\hline & \multicolumn{2}{|c|}{ Surgery } & \multicolumn{2}{|c|}{ Painful event } & \multicolumn{2}{|c|}{ Sad event } \\
\hline & $M$ & $S D$ & $M$ & $S D$ & $M$ & $S D$ \\
\hline \multicolumn{7}{|l|}{ Parent structure } \\
\hline Memory Question Elaboration (MQE) & 0.05 & 0.04 & 0.04 & 0.04 & 0.07 & 0.06 \\
\hline Yes-no Question Elaboration (YNE) & 0.26 & 0.10 & 0.25 & 0.12 & 0.26 & 0.11 \\
\hline Statement Elaboration (SE) & 0.13 & 0.09 & 0.17 & 0.15 & 0.16 & 0.10 \\
\hline Memory Question Repetition (MQR) & 0.11 & 0.06 & 0.08 & 0.07 & 0.12 & 0.08 \\
\hline Yes-no Question Repetition (YNR) & 0.25 & 0.09 & 0.25 & 0.12 & 0.17 & 0.09 \\
\hline Statement Repetition (SR) & 0.06 & 0.03 & 0.07 & 0.05 & 0.07 & 0.06 \\
\hline Evaluation (EVAL) & 0.15 & 0.07 & 0.15 & 0.09 & 0.16 & 0.09 \\
\hline \multicolumn{7}{|l|}{ Child structure } \\
\hline Memory Elaboration (ME) & 0.40 & 0.15 & 0.37 & 0.19 & 0.44 & 0.18 \\
\hline Memory Placeholder (MP) & 0.52 & 0.16 & 0.52 & 0.20 & 0.50 & 0.19 \\
\hline Off-topic Switch (OTS) & 0.09 & 0.10 & 0.10 & 0.15 & 0.07 & 0.08 \\
\hline \multicolumn{7}{|l|}{ Parent content } \\
\hline Positive Emotion (PEP) & 0.18 & 0.14 & 0.06 & 0.11 & 0.11 & 0.13 \\
\hline Negative Emotion (PEN) & 0.17 & 0.11 & 0.12 & 0.10 & 0.51 & 0.22 \\
\hline Neutral Emotion (PE_) & 0.15 & 0.11 & 0.07 & 0.09 & 0.10 & 0.12 \\
\hline Explanation (PEX) & 0.10 & 0.11 & 0.10 & 0.10 & 0.17 & 0.15 \\
\hline Coping (PCP) & 0.05 & 0.08 & 0.06 & 0.08 & 0.08 & 0.14 \\
\hline Pain $(\mathrm{PPN})$ & 0.19 & 0.12 & 0.49 & 0.25 & 0.03 & 0.10 \\
\hline Anxiety/Fear (PAF) & 0.16 & 0.09 & 0.11 & 0.09 & 0.08 & 0.11 \\
\hline \multicolumn{7}{|l|}{ Child content } \\
\hline Positive Emotion (CEP) & 0.22 & 0.24 & 0.04 & 0.11 & 0.13 & 0.21 \\
\hline Negative Emotion (CEN) & 0.14 & 0.17 & 0.10 & 0.14 & 0.34 & 0.33 \\
\hline Neutral Emotion (CE_) & 0.11 & 0.14 & 0.05 & 0.18 & 0.08 & 0.20 \\
\hline Explanation (CEX) & 0.22 & 0.21 & 0.18 & 0.29 & 0.30 & 0.31 \\
\hline Coping (CCP) & 0.03 & 0.07 & 0.05 & 0.11 & 0.07 & 0.19 \\
\hline Pain (CPN) & 0.15 & 0.19 & 0.36 & 0.33 & 0.04 & 0.15 \\
\hline Anxiety/Fear (CAF) & 0.11 & 0.13 & 0.07 & 0.12 & 0.06 & 0.12 \\
\hline Autonomy support & 3.03 & 0.25 & 3.05 & 0.30 & 3.14 & 0.30 \\
\hline
\end{tabular}

(memory question repetition [MQR], yes-no question repetition [YNR], and statement repetition [SR]) differently when reminiscing about sad events as compared with surgery or nonsurgery pain-related events. We hypothesized that children would mirror the reminiscing patterns of their parents.

\section{Content}

The content coding scheme was based on the research of Sales and colleagues (2003). Parent and child content were coded according to the following topics: emotion (negative, positive, neutral), anxiety/fear, explanations, coping, and pain. If utterances contained any words (i.e., any part of speech, e.g., noun, verb, adverb) related to one of the topics above, they were assigned an appropriate code. As a result, some utterances received more than one code (e.g., an utterance about being scared received two codes, i.e., negative emotion and anxiety/fear). Final proportions were calculated using the total number of content codes assigned. We expected that parents' and children's use of use content codes would differ when talking about sad versus painful events.

\section{Autonomy Support}

The coding scheme assessing parent support or control of child autonomy in the conversation was based on the research of Cleveland and colleagues (2005). Specifically, parental conversational turns were coded along a 5-point continuum, where 1 point was assigned to conversation-controlling utterances, and 5 points were assigned to utterances high on parent support of child autonomy in the conversation. Parental conversational turn was considered controlling if its function was to contradict the child's conversational turn or change the child's topic of conversation. Conversely, parental conversation turn was considered supportive of the child's autonomy if it functioned to agree with and encourage the child and/or follow in, sustain, and develop the child's conversation topic. We expected that parents would differ on the levels of autonomy support when discussing past sad versus painful events.

\section{Statistical Analyses}

Statistical analyses were conducted using the SPSS Version 24. Descriptive statistics, paired-sample $t$-tests, and repeated measures analysis of variance (ANOVA) 
were used to report sociodemographic characteristics of the sample and descriptive narrative variables (e.g., total number of utterances). For within-subject tests, the Huynh-Feldt estimate of sphericity was used to adjust $p$-values of $F$ tests (Maxwell \& Delaney, 2004). Frequency statistics were used to describe categorical variables (participant sex and ethnicity, household income, parent education level, and employment status). Means and standard deviations were reported for key variables. In line with previous research on parentchild reminiscing (Sales et al., 2003; Salmon \& Reese, 2015), each aspect of parent-child narratives (i.e., proportions of structural and content utterance codes) was analyzed using multivariate analysis of variance (MANOVA). Significant MANOVAs were followed up with omnibus tests (ANOVAs) and $t$-tests to identify specific differences between types of narratives. Differences in levels of parent autonomy support were analyzed using an omnibus test (ANOVA) with a series of follow-up $t$-tests. Bonferroni corrected alpha levels were used for follow-up $t$-tests. Power analysis indicated that in order for a .25 effect size to be detected with an $80 \%$ chance as significant at .05 alpha level, a sample of 39 parent-child dyads would be required ( $G^{*}$ Power; Faul, Erdfelder, Lang, \& Buchner, 2007).

\section{Results}

\section{Description of Events}

Two- to three-hours postsurgery, children reported their pain intensity as $3.14 / 10(S D=3.64)$ and their pain-related fear as $0.99 / 4(S D=1.45)$. Children reported higher levels of pain $(M=4.07 / 10 ; S D=$ 3.27 ) and approximately the same levels of painrelated fear $(M=0.84 / 4 ; S D=1.23)$ on the first day postsurgery. On average, the laboratory visit took place 0.54 months $(S D=0.15)$ postsurgery. For nonsurgery painful events, most children $(64 \%)$ nominated everyday pain experiences (i.e., minor injuries involving cuts and scrapes), $12 \%$ reminisced about getting a needle (e.g., flu shot), and $7 \%$ reminisced about a traumatic injury (e.g., breaking a limb). Children recalled high levels of pain intensity associated with these events $6.07 / 10(S D=3.87)$ and low to moderate levels of pain-related fear $(M=1.79 / 4$; $S D$ $=1.62)$. The painful event was reported to have taken place in the past year $(M=10.24$ months, $S D=$ 11.97 months).

For sad autobiographical events, the majority of children and parents $(23 \%)$ elected to talk about losing something important to them (e.g., a favorite toy), $17 \%$ of children reminisced about either someone (e.g., a relative or a pet) dying or about moving (e.g., to another city; $17 \%$ ). Children rated the average level of sadness of these events to be 2.28/7 ( $S D=1.82$; ' 1 ' $=$ very sad, ' 7 ' $=$ very happy). On average, the sad

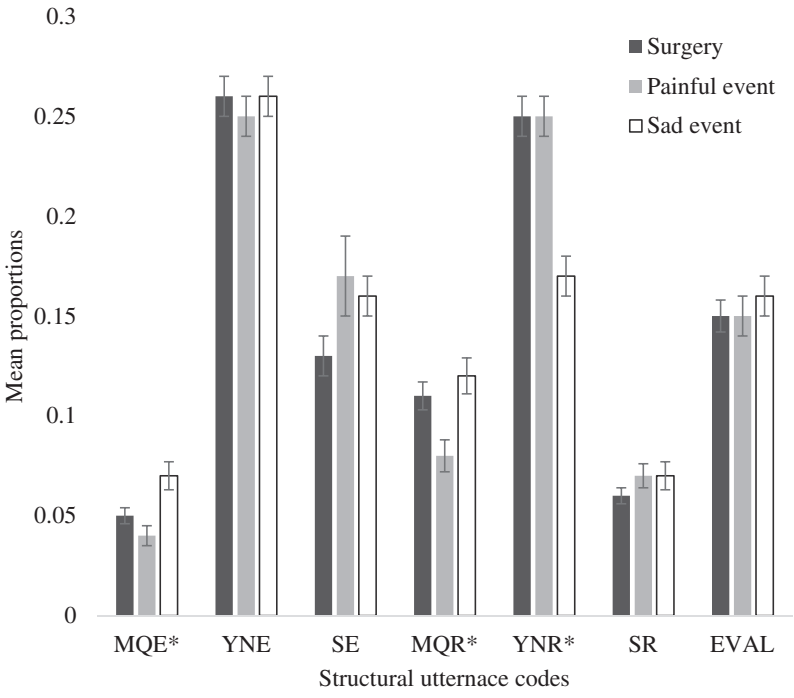

Figure 1. Parent structural reminiscing style. Error bars represent one standard error of the mean. MQE = memory question elaboration, $\mathrm{YNE}=$ yes-no question elaboration, $\mathrm{SE}=$ statement elaboration, $\mathrm{MQR}=$ memory question repetition, $\mathrm{YNR}=$ yes-no question repetition, $\mathrm{SR}=$ statement repetition, EVAL $=$ evaluation. ${ }^{*} p<.05$.

event took place in the previous $5.17(S D=8.80)$ months. Given the temporal discrepancy between the recent surgery and past autobiographical events, the subsequent MANOVAs controlled for when the events took place.

According to parents' ratings, they and their children reminisced significantly less about a nonsurgery painful event $(M=3.08 / 10, S D=2.50)$ as compared with a sad event $(M=5.01 / 10, S D=3.10 ; t[77]=$ $4.56, p<.001)$ or surgery $(M=5.04 / 10, S D=2.50$; $t[77]=5.39, p<.001)$ prior to the laboratory visit.

\section{Parental Reminiscing Style}

A MANOVA with three levels of the independent variable (type of event: surgery, sad event, and nonsurgery painful event) was conducted to examine differences in the structural utterance codes (MQEs, YNEs, SEs, MQRs, YNRs, SRs, and EVAL; Figure 1 ). A significant main effect was found for the type of structural utterance codes, $F(14,444)=3.97, p<$ .001. Follow-up omnibus tests indicated significant differences between types of utterances used by parents. Parents differed significantly in their use of elaborations (MQEs, $F[1.64,126.19]=9.93, p<$ $.001)$ and repetitions (MQRs, $F[1.87,144.31]=8.36$, $p<.001$; YNRs, $F(2,154)=26.59, p<.001)$. No significant differences between reminiscing topics were found for parent use of other types of elaborations (YNEs, SEs) or repetitions (SEs, EVALs, ps $>.05$ ). Parents used more MQEs when talking about a sad event $(M=0.07, S D=0.06)$ than when talking about surgery $(M=0.05, S D=0.04 ; t[77]=2.74, p=$ $.008)$ or when talking about the nonsurgery painful 
event $(M=0.04, S D=0.04 ; t[77]=3.78, p<.001)$. Parents used more MQEs when discussing surgery as compared with a nonsurgery painful event $(t[77]=$ $2.01, p=.048)$, however this difference was not significant at the adjusted alpha level of .017. When talking about a nonsurgery painful event $(M=0.08, S D=$ 0.07), parents used repetitions (MQR) less frequently as compared with recalling a sad event $(M=0.12, S D$ $=0.08 ; t[77]=3.55, p=.001)$ or surgery $(M=0.11$, $S D=0.06 ; t[77]=3.36, p=.001)$. Parents used yesno repetition questions (YNR) significantly less when reminiscing about a sad event $(M=0.17, S D=$ $0.09)$ as compared with reminiscing about surgery $(M=0.25, S D=0.09 ; t[77]=6.36, p<.001)$ or about the nonsurgery painful event $(M=0.25, S D=$ $0.12 ; t[77]=6.22, p<.001)$.

To summarize, when talking about sad events, parents used more elaborations and fewer repetitions than when talking about the surgery and nonsurgery painful event. Surgery and nonsurgery pain narratives differed only on the use of open-ended repetition questions.

\section{Children's Reminiscing Style}

An omnibus test was conducted to examine the differences in children's reminiscing style across three types of events. There were significant differences in children's use of memory elaborations $(F[2,154]=5.17$, $p=.007)$. Specifically, children tended to use more memory elaborations when talking about a sad event $(M=0.44, S D=0.18 ; t[77]=2.98, p=.004$, adjusted alpha level .017) than when talking about the nonsurgery painful event $(M=0.36, S D=0.19)$. Children used memory elaborations with equal frequency when talking about the surgery and nonsurgery painful events ( $p$ s $>.05$ ). Children's use of memory placeholders or off-topic switches did not differ by narrative type ( $p$ s $>.017)$.

\section{Parental Reminiscing Content}

A MANOVA with three levels of the independent variable (type of event: surgery, sad event, and nonsurgery painful event) was conducted to examine differences in the content utterance codes (type of content: PEP, PEN, PE, PEX, PCP, PPN, and PAF; Figure 2a). A significant main effect was found for the type of content codes $(F[14,442]=69.22, p<.001)$. Follow-up omnibus tests demonstrated significant differences in parent use of content categories. Specifically, parents differed in their use of words related to positive emotions $(F[1.81,135.39]=19.57$, $p<.001)$, negative emotions $(F[1.60,123.20]$ $=156.14, p<.001)$, neutral emotions $(F[2,154]=$ $15.25, p<.001)$, explanations $(F[1.76,135.28]=$ $11.33, p<.001)$, pain $(F[1.59,122.34]=144.70, p<$ $.001)$, and anxiety/fear $(F[2,154]=12.77, p<.001)$.
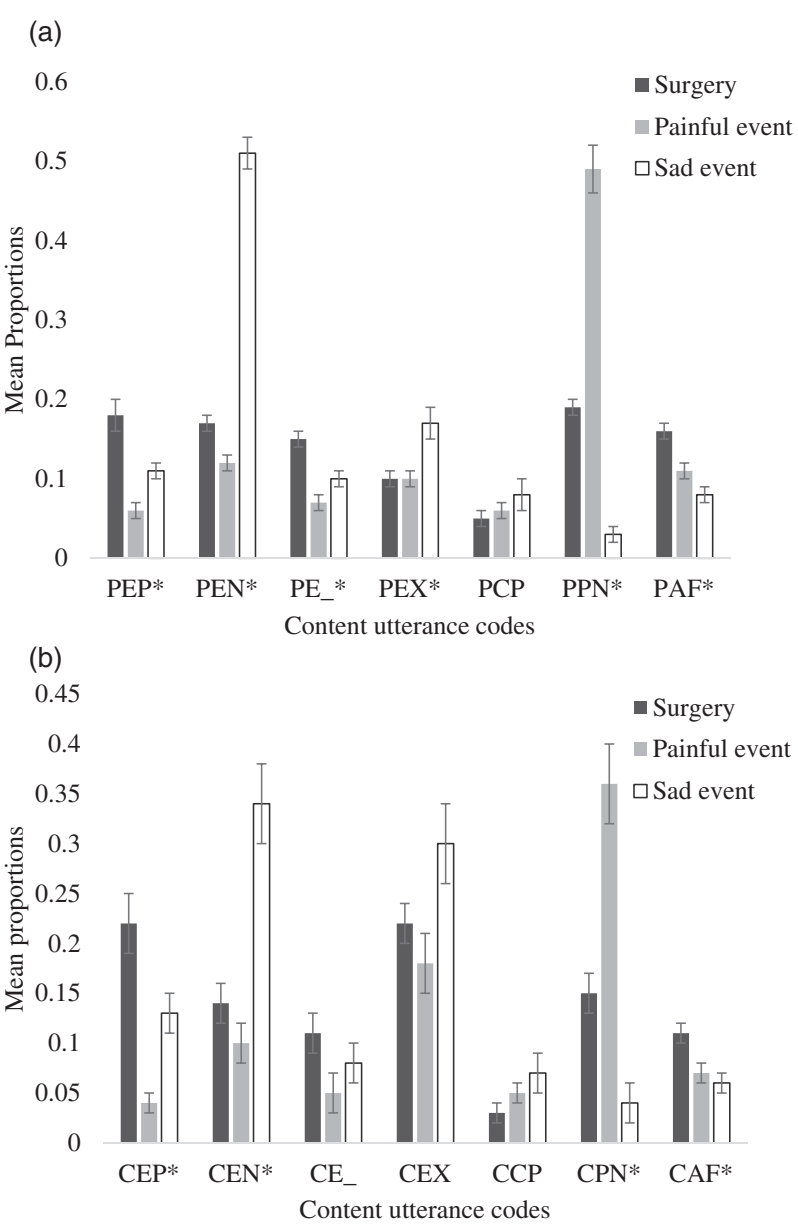

Figure 2. (a) Parent reminiscing content. Error bars represent one standard error of the mean. PEP $=$ parent positive emotion, $\mathrm{PEN}=$ parent negative emotion, $\mathrm{PE}$ - = parent neutral emotion, $\mathrm{PEX}=$ parent explanation, $\mathrm{PCP}=$ parent coping, $\mathrm{PPN}=$ parent pain-related words, $\mathrm{PAF}=$ parent anxiety and fear. ${ }^{*} p<.05$. (b) Child reminiscing content. Error bars represent one standard error of the mean. CEP = child positive emotion, $\mathrm{CEN}=$ child negative emotion, $\mathrm{CE}_{-}$ $=$ child neutral emotion, $\mathrm{CEX}=$ child explanation, $\mathrm{CCP}=$ child coping, $\mathrm{CPN}=$ child pain-related words, $\mathrm{CAF}=$ child anxiety and fear. ${ }^{*} p<.05$.

An adjusted alpha level of .017 was used for followup $t$-tests. No significant differences were found for parent use of words related to coping $(p>.05)$.

Specifically, parents used fewer positive emotionrelated words when talking about a nonsurgery painful event $(M=0.06, S D=0.11)$ as compared with surgery $(M=0.18, S D=0.14 ; t[77]=7.72, p<$ .001). Further, parents used fewer positive emotionrelated words when reminiscing about a sad event $(M$ $=0.11, S D=0.13)$ as compared with surgery $(t[77]=$ $3.35, p=.001)$. A similar pattern was found in the parental use of neutral emotion-related words when talking about surgery $(M=0.15, S D=0.11)$ as compared with a nonsurgery painful event $(M=0.07, S D=$ $0.09 ; t[77]=5.68, p<.001)$ or a sad event $(M=$ $0.10, S D=0.12 ; t[77]=3.13, p=.002)$. Negative 
emotion-related words were more frequently used in sadness narratives $(M=0.51, S D=0.22)$ as compared with surgery $(M=0.17, S D=0.11 ; t[77]=$ $12.61, p<.001)$ and nonsurgery painful event narratives $(M=0.12, S D=0.10 ; t[77]=14.43, p<.001)$. Moreover, parents used more negative emotionrelated words when talking about surgery than when talking about the nonsurgery painful event $(t[77]=$ $3.22, p=.002)$. However, surgery narratives $(M=$ $0.16, S D=0.09)$ contained more fear- and anxietyrelated words as compared with sadness narratives $(M$ $=0.08, S D=0.11 ; t[77]=4.91, p<.001)$ and nonsurgery painful event narratives $(M=0.11, S D=$ $0.09 ; t[77]=3.28, p=.002)$. Pain-related words were less frequently used in sadness narratives $(M=0.03$, $S D=0.10)$ as compared with surgery $(M=0.19, S D=$ $0.12 ; t[77]=8.49, p<.001)$ or nonsurgery painful event narratives $(M=0.49, S D=0.25 ; t[77]=14.79$, $p<.001)$. Furthermore, parents tended to use more pain-related words when talking about a nonsurgery painful event as compared with surgery $(t[77]=9.79, p$ $<.001)$. Finally, explanations were used more frequently when reminiscing about sadness $(M=0.17, S D$ $=0.15)$ as compared with surgery $(M=0.10, S D=$ $0.11 ; t[77]=3.43, p=.001)$ or the nonsurgery painful event $(M=0.10, S D=0.10 ; t[77]=4.14, p<.001)$.

To summarize, parents used more positive and neutral emotion-related words in surgery, as compared with sadness, narratives. Negative emotion-related words were used more frequently in sadness narratives than either of the painful narratives. Anxiety- and fear-related words occurred more often in the surgery but not sad event narratives. Parents employed the least amount of pain-related words in sadness narratives as compared with either of the painful events. Parents explained more when reminiscing about past sad, but not painful, events. Comparing surgery and nonsurgery painful narratives, parents used more emotional language when talking about surgery, whereas pain-related words were used more frequently in nonsurgery painful event narratives. The use of explanations did not differ across the painful narratives.

\section{Children's Reminiscing Content}

A MANOVA with three levels of the independent variable (type of event: surgery, sad event, and painful event) was conducted to examine differences in the child content utterance codes (type of content: CEP, $\mathrm{CEN}, \mathrm{CE}_{-}, \mathrm{CEX}, \mathrm{CCP}, \mathrm{CPN}$, and CAF; see Figure 2b). A significant main effect was found for the type of content codes $(F[14,442]=18.03, p<.001)$. Follow-up omnibus tests demonstrated significant differences in child use of content categories. Specifically, children differed in their use of words related to positive emotions $(F[1.81,139.02]=16.10, p<.001)$, negative emotions $(F[1.63,125.25]=156.14, p<$
$.001)$, explanations $(F[1.83,141.23]=3.30, p=$ $.037)$, pain $(F[1.66,127.67]=37.45, p<.001)$, and anxiety/fear $(F[2,154]=4.27, p=.016)$. An adjusted alpha level of .017 was used for follow-up $t$-tests. Children did not significantly differ in their use of words related to neutral emotions or coping ( $p$ s $>.05)$.

Positive emotion-related words were used more frequently when children reminisced about surgery $(M=$ $0.22, S D=0.24)$ as compared with a nonsurgery painful event $(M=0.04, S D=0.11 ; t[77]=5.98, p<$ $.001)$. However, children used more positive emotionrelated words when talking about a sad event $(M=$ $0.13, S D=0.21)$ than when talking about the nonsurgery painful event $(t[77]=3.31, p=.001)$. Negative emotion-related words were more frequently used in sadness $(M=0.34, S D=0.33)$ narratives as compared with surgery $(M=0.14, S D=0.17 ; t[77]=$ $4.88, p<.001)$ or nonsurgery painful event narratives $(M=0.10, S D=0.14 ; t[77]=6.22, p<.001)$. When reminiscing about surgery $(M=0.11, S D=0.13)$, children used more fear-/anxiety-related words as compared with sad event $(M=0.06, S D=0.12 ; t[77]$ $=2.78, p=.007)$ and nonsurgery painful event narratives $(M=0.07, S D=0.12 ; t[77]=2.22, p=.029)$. However, after applying the adjusted alpha, the latter difference was no longer significant. There were more explanations used in sadness $(M=0.30, S D=0.31)$ as compared with nonsurgery painful event narratives $(M=0.18, S D=0.29 ; t[77]=2.24, p=.028)$. After applying the adjusted alpha, the differences were no longer significant. Children used fewer pain-related words when reminiscing about a sad event $(M=0.04$, $S D=0.15)$ as compared with surgery $(M=0.15, S D$ $=0.19 ; t[77]=4.05, p<.001)$ or a nonsurgery painful event $(M=0.36, S D=0.33 ; t[77]=7.35, p<$ $.001)$. Nonsurgery painful event versus surgery narratives contained more pain-related words $(t[77]=$ $5.26, p<.001)$.

To summarize, children partially mirrored parent use of content codes. Specifically, children used more positive and less negative emotion-related words when reminiscing about surgery as compared with sad events. Anxiety/fear and pain-related words were less frequently used in sadness narratives as compared with either painful event narratives. Children used more emotional language (i.e., positive emotions) and less pain-related words when talking about surgery as compared with a nonsurgery related painful event.

\section{Parent Autonomy Support}

An omnibus ANOVA was conducted to examine differences in the levels of parental autonomy support across different narratives. Parents differed significantly on the levels of autonomy support across event types $(F[2,154]=3.36, p=.038)$. Follow-up $t$-tests 
revealed that parents were more autonomy supportive of their children when talking about sad events $(M=3.14, S D=0.30)$ as compared with talking about nonsurgery painful events $(M=3.05, S D=$ $0.30 ; t[77]=2.02, p=.048)$, or the surgery event $(M=3.03, S D=0.25 ; t[77]=2.30, p=.025)$. No significant differences were found for the levels of parent autonomy support in the surgery versus nonsurgery painful events $(p>.05)$.

\section{Associations Between the Recalled Event Characteristics and Utterance Types}

Children's report of postsurgery pain intensity was associated with the following utterance types: YNE $(r=$ $.22, p<.05)$, EVAL $(r=-.29, p<.05)$, and ME $(r=$ $-.26, p<.05)$. Children's postsurgery pain-related fear was not associated with parent or children use of any utterance types. The nonsurgical pain intensity was correlated with the parent use of EVAL $(r=.23$, $p<.05)$ and children's off-topic switch $(r=-.27$, $p<.05)$, as well as parent use of negative emotionrelated words $(r=.23, p<.05)$ and pain-related words $(r=-.33, p<.01)$. The nonsurgical painrelated fear was correlated with parent use of negative emotion-related words $(r=.24, p<.05)$.

\section{Associations Between the Individual Characteristics and Utterance Types}

A series of bivariate correlations and omnibus tests were conducted to examine the associations between participants' individual characteristics and reminiscing elements. Parent role and ethnicity were not significantly correlated with reminiscing styles ( $p$ s $>.05)$.

\section{Age}

Children's age was significantly associated with parent use of elaborations, such that parents used more elaborative elements (SE in surgery narratives, $r=.26, p<$ .05 ; MQE in sadness narratives, $r=.33, p<.01$ ) with older children. When reminiscing about the surgery, parents used negative emotion-related words more frequently with older children, $r=.26, p<.05$. When reminiscing about a nonsurgery painful event, parents used positive emotion-related words less frequently with older children, $r=-.23, p<.05$. Older children tended to talk more about coping with postsurgical pain, $r=.26, p<.05$.

\section{Children's Sex}

When reminiscing about past nonsurgery painful events, parents used elaborations (i.e., YNE) more frequently with boys $(M=0.28, S D=0.10)$ versus girls $(M=0.21, S D=0.13), F(1,79)=7.12, p<.01$. Parents talked about coping with nonsurgical pain more with boys $(M=0.06, S D=0.07)$ versus girls $(M$ $=0.03, S D=0.04), F(1,79)=4.19, p<.05$.

\section{Ethnicity}

Nonwhite children used more explanations when reminiscing about the surgery $(M=0.79, S D=2.06)$ as compared with white children $(M=0.18, S D=0.20)$, $F(1,81)=6.24, p<.05$. When talking about a past sad event, nonwhite children used more explanations $(M=0.70, S D=1.15)$ than white children $(M=$ $0.26, S D=0.28), F(1,78)=7.94, p<.01$. Children's age, sex, and ethnicity were not significantly associated with other reminiscing elements.

\section{Discussion}

This is the first study to examine differences in parent-child reminiscing about past painful events (i.e., postsurgical pain and a nonsurgery painful event) as compared with other autobiographical events involving negative emotions (i.e., sadness). Our findings revealed that parents talked about past events involving sadness versus pain differently. Specifically, when talking about past events involving sadness versus pain, parents used a more elaborative style (i.e., provided new contextual details, asked open-ended questions, used fewer repetitions) and children recalled more unique details. In terms of narrative content, parents and children provided more explanations and used more negative emotional (but not anxiety/fear related) words when reminiscing about past sad versus painful events. Finally, parents were more supportive of their children's autonomy in conversations about past sad versus painful events. These reminiscing elements (i.e., elaborations, explanations, and emotionladen language) have been linked to better developmental outcomes (e.g., language, autobiographical memory). Yet, more research is needed to elucidate if the differential reminiscing about past events involving pain versus sadness influence children's pain and health trajectories.

Through reminiscing about past negative events, parents create a fundamental learning environment for young children to process, make sense of, and react to their experiences (Nelson \& Fivush, 2004), including those involving distressing emotions and sensations. Parent-child reminiscing about past distressing experiences could be viewed as a type of scaffolding, such that parents provide and model a narrative account of their memory of the event and-if reminiscing in an elaborative, emotion-rich, and supportive way-can exemplify an adaptive approach for children to process these distressing experiences (Fivush et al., 2006). Moreover, reminiscing after the distressing event has ended is thought to be the ideal context for learning (Salmon \& Reese, 2016). When situational distress has passed, reminiscing allows for a unique opportunity to process and reframe any nonconstructive thoughts and feelings and formulate a positive context 
for future coping (Sales \& Fivush, 2005). While the field of pediatric pain has devoted extensive research attention to parent-child interactions within the immediate painful context (e.g., vaccinations, needle procedures; McMurtry, Chambers, McGrath, \& Asp, 2010; Schechter et al., 2007), far less is known about how these salient events are subsequently remembered, discussed, and coconstructed in memory. Events involving sadness and pain are both emotionally distressing and frequently experienced; however, events involving past pain generate a different narrative account. For instance, in a study of parent-child everyday conversations, pain was mentioned quite frequently $(29 \%$ of transcripts contained at least one pain-related word; Craig, Stanford, Fairbairn, \& Chambers, 2006). However, with regard to the temporal context of the painful event, the conversations were about present $(32 \%)$ or imaginary $(12 \%)$ pain, whilst past pain was discussed merely once $(2 \%$; Craig et al., 2006). Coupled with our current findings, this raises intriguing questions about the fundamental experience of pain and what it is that makes parents reminisce with their children about past pain differently.

Early experiences involving both pain and sadness are distressing; however, there are fundamental differences between these events that may explain the differences in reminiscing found in the current study. By its very nature, the immediate experience of pain monopolizes attention (Eccleston \& Crombez, 1999). However, once the sensation of pain subsides, the distressing emotional aspects of the experience are likely to be avoided; yet, pain is rarely forgotten. To contrast this with sadness, while distressing emotions do not necessarily and immediately command our attention, these events are more likely to be discussed in more detail, more often, and elicit more empathic and supportive responses postfactum (Cassano, Zeman, \& Sanders, 2014). Moreover, socially accepted coping and relief strategies for events involving pain versus sadness differ (Cassano et al., 2014; Walker \& Zeman, 1992). Acute pain elicits an immediate response from caregivers that usually involves medication and/or soothing and distracting behaviors (Chau \& Koren, 2014; Taddio, 2014; Taddio et al., 2015). Parents' empathic responses to procedural pain in children were associated with increased levels of children's distress (Blount et al., 1997). Sadness, on the other hand, produces a range of empathic responses that often extend far beyond the immediate situation (Fivush, Berlin, et al., 2003). Helping a child effectively cope with a sad event typically involves discussing the event on multiple occasions to assist the child in making sense of the experience, fostering empathy and sense of self, and showing affection (Fivush, Berlin, et al., 2003; Fivush, Hazzard, McDermott Sales, Sarfati, \& Brown, 2003; Laible \& Song, 2006).
Indeed, our findings revealed that parents reported talking to children more often about past events involving sadness versus pain and thus, may have less experience reminiscing about painful events. This may partially explain why children are more readily equipped to empathically respond to negative emotions, as compared with pain, earlier in development (Bandstra et al., 2011).

We hypothesize that parents may also believe that talking about painful events with children may itself be maladaptive by evoking a sensation of pain or contributing to pain-related fear and avoidance. In contrast, although reminiscing about negative emotions like sadness often evokes re-experiencing those emotions, parents may believe that reminiscing about sad events enables children to process and overcome that emotion. Furthermore, discussing these types of events may evoke different emotions in parents themselves; talking to children about their pain experiences may simply be more distressing than talking to them about sadness. Future research should examine parental beliefs about, and distress evoked by, reminiscing with children about past painful versus sad events.

Individual differences were revealed in parents' and children's use of narrative codes, such that older children discussed coping with postsurgical pain more frequently, and parents of older children elaborated more when talking about the surgery and a past event involving sadness. Parents of older children also used more negative emotional language when reminiscing about the surgery and fewer words associated with positive emotions when discussing a nonsurgical painful event. These findings are in line with the previous research that found parents to be more repetitive with younger children and using more emotional language with older children (Sales et al., 2003). With regard to sex differences and similar to previous research (Sales et al., 2003), when reminiscing about past painful events, parents elaborated and talked more about pain-related coping with boys versus girls. These findings are preliminary, yet they may be indicative of a potential social process underlying sex differences in acute and chronic pediatric pain (Fowler-Kerry \& Lander, 1991; Huguet et al., 2017). Further, sex differences were revealed only for narratives about past nonsurgical painful events but not postsurgical painful or sad events. In previous studies, parents tended to elaborate more when reminiscing with girls versus boys about past distressing events (e.g., fear, anger, and sadness; Fivush, Berlin, et al., 2003). Past events involving everyday pain as opposed to a surgery (i.e., an experience that, in addition to being painful, is fear- and/or anxiety-provoking) or an emotionally distressing experience (i.e., one involving sadness) may evoke unique sex-specific narratives that warrant further examination. Future research should examine sex 
differences in parent-child reminiscing in relation to developmental and health outcomes. Finally, nonwhite children, compared with white children who comprised the majority $(84 \%)$ of the sample, used more explanations when reminiscing about past events that involved sadness and surgery. While preliminary, this finding is contrary to the previous studies that demonstrated Euro-American children providing more explanations compared with Chinese children (Wang, Doan, \& Song, 2010).

Children's postsurgical pain intensity was associated with parent reminiscing style, such that more frequent parent use of close-ended elaborative questions was related to higher ratings of postsurgical pain, whereas parent use of evaluations and children's recall of unique details about the surgery was associated with lower ratings of postsurgical pain. Significant correlations between children's nonsurgical pain and parent reminiscing style followed a different pattern. Specifically, higher levels of nonsurgical pain intensity were related to parents' more frequent use of evaluations and negative emotional language and less frequent use of pain-related words. Nevertheless, these findings are preliminary and may be confounded by the timing of ratings (i.e., children rated their nonsurgical pain and fear and affect associated with the sad event after reminiscing about the events), instruments used to obtain the ratings (i.e., the CFS and the emotions faces scale), as well as the order effects discussed below.

The results of this study should be viewed in light of limitations and highlight avenues for future research. First, parent-child narratives were elicited within a laboratory setting as opposed to a more naturalistic environment (e.g., home), which could have limited the ecological validity of the observed interactions. Further, the laboratory where children were tested is located in the same hospital where the tonsillectomy took place, therefore, the hospital setting may have more readily evoked children's memories for the surgery and contributed to the differences between parent-child narratives. Second, although some individual differences that may have influenced reminiscing styles (i.e., children's age, sex, and ethnicity) were examined, future research should examine other factors such as parent anxiety, parenting style, and painrelated cognitions on parent-child reminiscing styles. The influence of reminiscing style on children's painrelated beliefs and cognitions represents an important avenue for future research. Third, only two types of distressing events (i.e., pain and sadness) were compared. Given the core role of threat in prevailing theories of pain (Asmundson, Noel, Petter, \& Parkerson, 2012; Eccleston \& Crombez, 1999), future research should compare parent-child narratives about events involving threat (e.g., getting lost) with events involving pain. Furthermore, the nonsurgery painful event and the past event involving sadness were not limited by any specific timeline or event type. Thus, elicited events ranged from occurring very recently to being more removed in time and from minor (e.g., watching a sad movie, getting a bruise) to major (e.g., death of a family member, breaking a limb) life events. While children in the study experienced the tonsillectomy surgery only once, the other nonsurgery painful events (e.g., needles) were likely repeatedly experienced. Research suggests that memory for repeated versus single events is worse and more susceptible to distortion by postevent information provided by others (Powell \& Thomson, 1996). The same order of conversation topics may have contributed to the differences between narratives. In previous studies that employed a similar design, parents were most elaborative when talking about the last conversation topic (e.g., fear in Fivush, Berlin, et al, 2003). In the present study, a similar pattern emerged, such that parents were more elaborative when reminiscing about sadness (i.e., the last conversation topic). Future research should counterbalance reminiscing topics and incorporate a warm-up task for parents and children to encourage dyads to reminisce as naturally and openly as they normally would outside of the laboratory setting. Another possible limitation that pertains to the elicited events concerns the direct versus vicarious experience, such that children directly experienced the postsurgical and everyday pain with their parents observing those experiences. Conversely, some of the elicited sad events and the associated sad affect (e.g., from a pet dying) were directly experienced by both, parents and children. This difference may have resulted in how parents talked about sad events. Although the importance and benefits of elaborative, emotion-rich reminiscing have been investigated in diverse samples (Peterson, Jesso, \& McCabe, 1999), parent reminiscing styles about past events involving pain versus sadness in families of more diverse sociocultural backgrounds needs further examination. It should also be noted that some of the instruments used in the study (i.e., the CFS and the emotion faces scale) were not validated for use with children younger than 5 years. Finally, the cross-sectional nature of the present study precludes any inferences with regard to changes in parent reminiscing style over time or the relationship of reminiscing to child outcomes. Future research should also employ sequential dyadic analyses to examine nuances of parent-child reminiscing (e.g., who initiates more elaborative style elements) and whether it differs across reminiscing topics.

In conclusion, this is the first study to examine parent-child reminiscing about past painful events and how this differs from other emotionally distressing events involving sadness. Findings revealed that 
when reminiscing about past sad, but not painful, events, parents and children used narrative elements (i.e., elaborations, emotion-laden language) that have been linked to better developmental outcomes. More research is needed to examine whether this differential reminiscing influences pain-related cognitions (e.g., children's memory for pain) as well as their healthrelated behaviors (e.g., medical adherence) and future pain experiences.

\section{Supplementary Data}

Supplementary data can be found at: https://academic.oup. com/jpepsy.

\section{Funding}

This work was supported by the Society of Pediatric Psychology Targeted Research Grant, American Pain Society Future Leader in Pain Grant, Maternal Newborn Child, and Youth Strategic Clinical Network Health Outcomes Improvement Fund, and University of Calgary Faculty of Science Seed Grant was awarded to M. Noel. M. Noel was supported the Vi Riddell Pediatric Pain Initiative and the Alberta Children' Hospital Research Institute. M. Pavlova was a recipient of the 2017 Alberta SPOR Graduate Studentship in Patient-Oriented Research, which is jointly funded by Alberta Innovates and the Canadian Institutes of Health Research. S.A. Graham' s contribution was supported by funding from the Alberta Children's Hospital Foundation.

Conflicts of interest: None declared.

\section{References}

Asmundson, G. J., Noel, M., Petter, M., \& Parkerson, H. A. (2012). Pediatric fear-avoidance model of chronic pain: Foundation, application and future directions. Pain Research \& Management, 17, 397-405.

Bandstra, N. F., Chambers, C. T., McGrath, P. J., \& Moore, C. (2011). The behavioural expression of empathy to others' pain versus others' sadness in young children. Pain, 152, 1074-1082.

Blount, R. L., Cohen, L. L., Frank, N. C., Bachanas, P. J., Smith, A. J., Manimala, M. R., \& Pate, J. T. (1997). The Child-Adult Medical Procedure Interaction Scale-Revised: An assessment of validity. Journal of Pediatric Psychology, $22,73-88$.

Cassano, M. C., Zeman, J. L., \& Sanders, W. M. (2014). Responses to children's sadness: Mothers' and fathers' unique contributions and perceptions. MErrill-Palmer Quarterly, 60, 1-23.

Castilla, D., Botella, C., García-Palacios, A., Farfallini, L., \& Miralles, I. (2012). Experience-sampling methodology with a mobile device in fibromyalgia. International Journal of Telemedicine and Applications, 2012, 162673.

Chau, K., \& Koren, G. (2014). Principles of pain pharmacology in paediatrics. In P. J. McGrath, B. Stevens, S. M. Walker, \& W. T. Zempsky (Eds.), Oxford textbook of paediatric pain, pp. 429-435. New York, NY: Oxford University Press.

Cleveland, E. S., \& Morris, A. (2014). Autonomy support and structure enhance children's memory and motivation to reminisce: A parental training study. Journal of Cognition and Development, 15, 414-436.

Cleveland, E. S., \& Reese, E. (2005). Maternal structure and autonomy support in conversations about the past: Contributions to children's autobiographical memory. Developmental Psychology, 41, 376-388.

Craig, K. D., Stanford, E. A., Fairbairn, N. S., \& Chambers, C. (2006). Emergent pain language communication competence in infants and children. Enfrance, 58, 52-71.

Eccleston, C., \& Crombez, G. (1999). Pain demands attention: A cognitive-affective model of the interruptive function of pain. Psychological Bulletin, 125, 356-366.

Faul, F., Erdfelder, E., Lang, A. G., \& Buchner, A. (2007). G*Power 3: A flexible statistical power analysis program for the social, behavioral, and biomedical sciences. Behavior Research Methods, 39, 175-191.

Fivush, R., Berlin, L. J., Sales, J. M., Mennuti-Washburn, J., \& Cassidy, J. (2003). Functions of parent-child reminiscing about emotionally negative events. Memory, 11, 179-192.

Fivush, R., \& Fromhoff, F. A. (1988). Style and structure in mother child conversations about the past. Discourse Processes, 11, 337-355.

Fivush, R., Haden, C. A., \& Reese, E. (2006). Elaborating on elaborations: Role of maternal reminiscing style in cognitive and socioemotional development. Child Development, $77,1568-1588$.

Fivush, R., Hazzard, A., McDermott Sales, J., Sarfati, D., \& Brown, T. (2003). Creating coherence out of chaos? Children's narratives of emotionally positive and negative events. Applied Cognitive Psychology, 17, 1-19.

Fowler-Kerry, S., \& Lander, J. (1991). Assessment of sex differences in children's and adolescents' self-reported pain from venipuncture. Journal of Pediatric Psychology, 16, 783-793.

Hicks, C. L., von Baeyer, C. L., Spafford, P. A., van Korlaar, I., \& Goodenough, B. (2001). The Faces Pain ScaleRevised: Toward a common metric in pediatric pain measurement. Pain, 93, 173-183.

Huguet, A., Olthuis, J., McGrath, P. J., Tougas, M. E., Hayden, J. A., Stinson, J. N., \& Chambers, C. T. (2017). Systematic review of childhood and adolescent risk and prognostic factors for persistent abdominal pain. Acta Paediatrica, 106, 545-553.

Hyland, E. J., D'Cruz, R., Harvey, J. G., Moir, J., Parkinson, C., \& Holland, A. J. A. (2015). An assessment of early Child Life Therapy pain and anxiety management: A prospective randomised controlled trial. Burns, 41, 1642-1652.

Kain, Z. N., Mayes, L. C., Caldwell-Andrews, A. A., Karas, D. E., \& McClain, B. C. (2006). Preoperative anxiety, postoperative pain, and behavioral recovery in young children undergoing surgery. Pediatrics, 118, 651-658.

Laible, D. (2004). Mother-child discourse in two contexts: Links with child temperament, attachment security, and socioemotional competence. Developmental Psychology, 40, 979-992. 
Laible, D., \& Song, J. (2006). Constructing emotional and relational understanding: The role of affect and motherchild discourse. Merrill-Palmer Quarterly-Journal of Developmental Psychology, 52, 44-69.

Leyva, D., Berrocal, M., \& Nolivos, V. (2014). Spanishspeaking parent-child emotional narratives and children's social skills. Journal of Cognition and Development, 15, 22-42.

Leyva, D., \& Nolivos, V. (2015). Chilean family reminiscing about emotions and its relation to children's selfregulation skills. Early Education and Development, 26, 770-791.

MacLaren, J. E., \& Kain, Z. N. (2008). Prevalence and predictors of significant sleep disturbances in children undergoing ambulatory tonsillectomy and adenoidectomy. Journal of Pediatric Psychology, 33, 248-257.

Maxwell, S. E., \& Delaney, H. D. (2004). Designing experiments and analyzing data: A model comparison perspective (2nd edn). Mahwah, NJ: Lawrence Erlbaum Associates.

McMurtry, C. M., Chambers, C. T., McGrath, P. J., \& Asp, E. (2010). When "don't worry" communicates fear: Children's perceptions of parental reassurance and distraction during a painful medical procedure. Pain, 150, 52-58.

McMurtry, C. M., Noel, M., Chambers, C. T., \& McGrath, P. J. (2011). Children's fear during procedural pain: Preliminary investigation of the Children's Fear Scale. Health Psychology, 30, 780-788.

Nelson, K., \& Fivush, R. (2004). The emergence of autobiographical memory: A social cultural developmental theory. Psychological Review, 111, 486-511.

Noel, M., Chambers, C. T., McGrath, P. J., Klein, R. M., \& Stewart, S. H. (2012). The influence of children's pain memories on subsequent pain experience. Pain, 153, 1563-1572.

Noel, M., Palermo, T. M., Chambers, C. T., Taddio, A., \& Hermann, C. (2015). Remembering the pain of childhood: Applying a developmental perspective to the study of pain memories. Pain, 156, 31-34.

Noel, M., Rabbitts, J. A., Fales, J., Chorney, J., \& Palermo, T. M. (2017). The influence of pain memories on children's and adolescents' post-surgical pain experience: A longitudinal dyadic analysis. Health Psychology, 36, 987-995.

Peterson, C. (2015). A decade later: Adolescents' memory for medical emergencies. Applied Cognitive Psychology, 29, 826-834.
Peterson, C., Jesso, B., \& McCabe, A. (1999). Encouraging narratives in preschoolers: An intervention study. Journal of Child Language, 26, 49-67.

Powell, M. B., \& Thomson, D. M. (1996). Children's memory of an occurrence of a repeated event: Effects of age, repetition, and retention interval across three question types. Child Development, 67, 1988-2004.

Reese, E., \& Robertson, S. J. (2019). Origins of adolescents' earliest memories. Memory, 27, 79-91.

Sales, J. M., \& Fivush, R. (2005). Social and emotional functions of mother-child reminiscing about stressful events. Social Cognition, 23, 70-90.

Sales, J. M., Fivush, R., \& Peterson, C. (2003). Parental reminiscing about positive and negative events. Journal of Cognition and Development, 4, 185-209.

Salmon, K., \& Reese, E. (2015). Talking (or not talking) about the past: The influence of parent-child conversation about negative experiences on children's memories. Applied Cognitive Psychology, 29, 791-801.

Salmon, K., \& Reese, E. (2016). The benefits of reminiscing with young children. Current Directions in Psychological Science, 25, 233-238.

Schechter, N. L., Zempsky, W. T., Cohen, L. L., McGrath, P. J., McMurtry, C. M., \& Bright, N. S. (2007). Pain reduction during pediatric immunizations: Evidence-based review and recommendations. Pediatrics, 119, e1184-e1198.

Taddio, A. (2014). Needle procedures. In P. J. McGrath, B. Stevens, S. M. Walker, \& W. T. Zempsky (Eds.), Oxford textbook of paediatric pain, pp. 184-193. New York, NY: Oxford University Press.

Taddio, A., McMurtry, C. M., Shah, V., Riddell, R. P., Chambers, C. T., Noel, M., ... HELPinKids\&Adults Team. (2015). Reducing pain during vaccine injections: Clinical practice guideline. CMAJ, 187, 975-982.

Valentino, K., Hibel, L. C., Cummings, E. M., Nuttall, A. K., Comas, M., \& McDonnell, C. G. (2015). Maternal elaborative reminiscing mediates the effect of child maltreatment on behavioral and physiological functioning. Development and Psychopathology, 27, 1515-1526.

Walker, L. S., \& Zeman, J. L. (1992). Parental response to child illness behavior. Journal of Pediatric Psychology, 17, 49-71.

Wang, Q., Doan, S. N., \& Song, Q. (2010). Talking about internal states in mother-child reminiscing influences children's self-representations: A cross-cultural study. Cognitive Development, 25, 380-393. 